\title{
Grade of recurrent in situ and invasive carcinoma following treatment of pure ductal carcinoma in situ of the breast
}

\author{
RR Millis', SE Pinder,2, K Ryder ${ }^{3}$, R Howitt $^{4}$ and SR Lakhani ${ }^{5}$ \\ 'Hedley Atkins Cancer Research UK Breast Pathology Laboratory, Guy's Hospital, London SEI 9RT, UK; '2Department of Histopathology, University of \\ Nottingham, Nottingham City Hospital, Hucknal Road, Nottingham NG5 IPB, UK; ${ }^{3}$ Academic Oncology Unit, Guy's Hospital, London SEI 9RT, UK; \\ ${ }^{4}$ Department of Cellular Pathology, Southampton General Hospital, Southampton SO 6 6YD, UK; ${ }^{5}$ The Breakthrough Toby Robins Breast Cancer \\ Research Centre, ICR and the Royal Marsden Hospital, London SW3 6JB, UK
}

The grade of recurrent in situ and invasive carcinoma occurring after treatment of pure ductal carcinoma in situ (DCIS) has been compared with the grade of the original DCIS in 122 patients from four different centres (The Royal Marsden Hospitals, London and Sutton, 57 patients; Guy's Hospital, London, 19 patients; Nottingham City Hospital, 3 I patients and The Royal Liverpool Hospital, I5 patients). The recurrent carcinoma was pure DCIS in 70 women (57\%) and in 52 women (43\%) invasive carcinoma was present, which was associated with an in situ element in 43. In all, 19 patients developed a second recurrence (pure DCIS in II and invasive with or without an in situ element in eight). The majority of invasive carcinomas followed high-grade DCIS. There was strong agreement between the grade of the original DCIS and that of the recurrent DCIS $(\kappa=0.679)$, which was the same in 95 of 1 I 3 patients (84\%). The grade of the original DCIS showed only fair agreement with the grade of recurrent invasive carcinoma $(\kappa=0.24 \mathrm{I})$, although agreement was stronger with the pleomorphism score of the recurrent carcinoma $(\kappa=0.396)$. There was moderate agreement, in recurrent invasive lesions, between the grade of the DCIS and that of the associated invasive element $(\kappa=0.5 \mathrm{I} 5)$. Other features that showed moderate or strong agreement between the original and recurrent DCIS were necrosis and periductal inflammation. The similarity between the histological findings of the original and subsequent DCIS is consistent with the concept that recurrent lesions represent regrowth of residual carcinoma. In addition, although agreement between the grade of the original DCIS and that of any subsequent invasive carcinoma was only fair, there is no suggestion that low-grade DCIS lesions progress to higher grade lesions or to the development of higher grade invasive carcinoma. This is in agreement with immunohistochemical and molecular data indicating that low-grade and high-grade mammary carcinomas are quite different lesions. British Journal of Cancer (2004) 90, 1538-1542. doi:I0.1038/sj.bjc.660I704 www.bjcancer.com

Published online 30 March 2004

(c) 2004 Cancer Research UK

Keywords: breast carcinoma; ductal carcinoma in situ (DCIS); histological grade

The best treatment for ductal carcinoma in situ (DCIS) of the breast has yet to be determined, although there is an increasing body of evidence that radiotherapy is of benefit after wide local excision (Julien et al, 2000; UK Coordinating Committee on Cancer Research (UKCCCR) Ductal Carcinoma in situ Working Party, 2003). The increase in the number of cases diagnosed since the introduction of mammographic screening has emphasised the considerable diversity exhibited by these lesions. Several histopathological classifications have been proposed during the past two decades (Lagios et al, 1989; Holland et al, 1994; Poller et al, 1994; Silverstein et al, 1995, 1996; Scott et al, 1997; Sloane et al, 1998). These are all based primarily on nuclear morphology and also, in some, on the presence or absence of necrosis or cell polarisation. The histological type, together with the lesion size, and, perhaps of most significance, the size of the margin of clearance have been found to be the best determinants of the risk of recurrence (Lagios et al, 1989; Poller and Ellis, 1995; Silverstein et al, 1996, 1999; Bijker et al, 2001a; Douglas-Jones et al, 2002).

*Correspondence: Dr SE Pinder; E-mail: sarah.pinder@nottingham.ac.uk Received 20 October 2003; accepted 19 January 2004; published online 30 March 2004
Approximately half of the recurrent lesions remain in situ, but half show evidence of invasion. Owing to the potential for metastasis, the latter are of considerable significance as the chance of successful eradication of the disease may have been lost. In several studies the incidence of recurrence, at least in the short term, has been found to be higher following high-grade (poorly differentiated) DCIS than following low-grade (well differentiated) lesions (Lagios et al, 1989; Schwartz et al, 1992; Bellamy et al, 1993; Solin et al, 1993; Sneige et al, 1995; Silverstein et al, 1996; Fisher et al, 1999). Moreover, in some studies, the incidence of an invasive recurrence has been found to be higher after high-grade DCIS than after low-grade lesions (Lagios et al, 1989; Bellamy et al, 1993). This suggests that more radical initial treatment may be indicated for high-grade DCIS than for low-grade DCIS.

Histopathological studies of invasive carcinoma with an associated in situ component have shown a close link between the grade of the in situ and that of the invasive component (Lampejo et al, 1994; Goldstein and Murphy, 1996; Gupta et al, 1997). Furthermore, the clinical outcome is related to the histology of the DCIS. Those with a well-differentiated in situ component associated with their invasive carcinoma have a more favourable prognosis than patients with poorly differentiated lesions (Lampejo et al, 1994; Gupta et al, 1997). Studies of recurrent in situ and 
invasive carcinoma following treatment for pure in situ carcinoma have shown a high degree of correlation between the grades of the original and recurrent tumours (Barnes et al, 2000; Bijker et al, $2001 b$ ). If recurrent lesions following pure DCIS are always of the same grade, it can be argued that low-grade DCIS is not only less likely to recur and to develop invasive disease but also that, if it does recur, the chance of successful treatment is still there. If this is so, such lesions could be managed in a less radical manner.

The present study was undertaken to try to establish the nature of recurrent lesions, both in situ and invasive, occurring following the treatment of pure DCIS.

\section{MATERIALS AND METHODS}

The files of patients treated for DCIS were reviewed and those in whom a recurrent carcinoma had occurred after an interval of at least 6 months from initial treatment were selected. The recurrent lesions were within the ipsilateral residual breast or, in a few cases, within the mastectomy scar. Only cases in which the slides of both the primary and recurrent tumour were available for review were included in the study. The 122 patients studied were seen and treated at the Royal Marsden Hospital, London and Surrey (RMH) (57 patients; initial diagnosis between 1975 and 1990), Guy's Hospital, London (Guy's) (19 patients; initial diagnosis 19751994), Nottingham City Hospital (NCH) (31 patients; initial diagnosis 1975-1996) and the Royal Liverpool Hospital (RLH) (15 patients; initial diagnosis 1982-1996). Preliminary findings relating to some of the $\mathrm{NCH}$ patients have been included in previous presentations (Barnes et al, 2000).

The age of the patients ranged from 24 to 81 years (Guy's patients, 24-81 years, median 54 years; RMH patients, 28-72 years, median 52 years; $\mathrm{NCH}$ patients, $29-75$ years, median 52 years; RLH patients, 37-64 years, median 54 years). Treatment in the majority of cases was surgical excision either alone or in combination with adjuvant tamoxifen or radiotherapy or both. In a minority, initial treatment was simple or radical mastectomy. The time to first recurrence ranged from 6 to 177 months (Guy's patients, 18-177 months, median 69 months; RMH patients, 7125 months, median 39 months; NCH patients, 6 months -143 months, median 56 months; RLH patients, 12-180 months, median 36 months).

The following features were recorded in the primary in situ tumours: nuclear grade, architectural pattern, necrosis and periductal fibrosis and inflammatory (lymphoplasmacytic) reaction. In the recurrent lesions, the nuclear grade of the in situ carcinoma and, in most cases, also the presence of necrosis, the architectural pattern and periductal changes were noted, as was the grade of the invasive lesion where present. The slides were reviewed separately by two of the authors in all cases, and where there was disagreement they were then studied together or in some cases a third author was consulted.

The nuclear grade of the in situ lesions was based on the features used in the United Kingdom National Health Service Breast Screening Programme (UK NHSBSP) and the European Commission Working Group on Breast Screening Pathology (Sloane et al, 1998). High-grade DCIS is composed of cells with pleomorphic nuclei, which are usually large, show marked variation in size and shape, have coarse chromatin and prominent nucleoli and are irregularly spaced. Mitoses are usually frequent and both individual cell necrosis and phagocytosis may be present. Lowgrade DCIS consists of cells with monomorphic nuclei that are usually small, spherical and central. Nucleoli are inconspicuous and the chromatin is uniform. Mitoses are rare, as is individual cell necrosis. Intermediate grade DCIS is composed of cells that have intermediate features and cannot readily be assigned to either of the above categories. There is mild to moderate pleomorphism that is less than in high-grade lesions, but the monotony of low-grade
DCIS is absent and nucleoli are often present. In lesions where differing ducts showed variation in nuclear grade, all grades present were recorded. Architectural pattern was described as solid, cribriform, micropapillary or intracystic papillary. When more than one pattern was present, each was recorded. Necrosis was scored as 0 absent, 1 mild, 2 moderate or 3 marked (usually of comedo type). Periductal fibrosis and inflammatory reaction were both scored as 1 absent/mild, 2 moderate or 3 marked. The grade of recurrent invasive carcinoma was based on the Nottingham modification of Bloom and Richardson grading (Elston and Ellis, 1991).

\section{RESULTS}

The grade of the DCIS in the original lesions is shown in Table 1. Over $75 \%$ of cases were high grade and only eight cases $(6.5 \%)$ of pure low-grade DCIS were present in the study.

At first recurrence, $70(57 \%)$ cases recurred as pure DCIS and 52 (43\%) contained invasive carcinoma. Of those with invasion, 43 recurred as invasive with an associated in situ component and nine as invasive without an in situ component identified. One of the invasive lesions with an in situ component was micro-invasive ( $<1 \mathrm{~mm}$ in maximum dimension) and is not included in any further analysis of invasive tumours. As shown in Table 1, in the 113 cases in which DCIS was present in the first recurrence, when the highest DCIS grade of both the first and recurrent lesion were considered, there was close agreement between the grades $(\kappa=0.679)$. The grades were the same in 95 of 113 cases $(84 \%)$. The first lesion contained more than one grade of DCIS in 13 cases and in seven cases there was more than one grade in the second DCIS (see Table 2). If all grades were considered, there were nine cases in which the grade of the second DCIS was not seen in the first lesion. In seven, the grade was lower and in two it was higher.

In all, 19 patients had a second recurrence. In nine of these patients, both recurrences were pure in situ, in four both contained in situ and invasive disease, in four the first was pure in situ but the second contained invasive carcinoma and in two patients, although invasion was present in the first recurrence, it could not be identified in the second. Again, there was close agreement between the grade of the DCIS in the original lesion and the second recurrence (data not shown).

The majority of the invasive recurrences followed high-grade DCIS (42 of 55; 76\%) and only four occurred in patients in whom

Table I Comparison of original and recurrent DCIS grades

\begin{tabular}{|c|c|c|c|c|}
\hline \multirow[b]{2}{*}{$\begin{array}{l}\text { Original DCIS grade } \\
\text { (highest present) }\end{array}$} & \multicolumn{4}{|c|}{ Recurrent DCIS grade (highest present) ${ }^{a}$} \\
\hline & $\mathbf{I}$ & 2 & 3 & Total \\
\hline I & 6 & 1 & 1 & 8 \\
\hline 2 & 2 & 16 & 2 & 20 \\
\hline 3 & 1 & 11 & 73 & 85 \\
\hline Total & 9 & 28 & 76 & 113 \\
\hline
\end{tabular}

${ }^{\mathrm{a} O n l y}$ first recurrence included. $\chi^{2}=97.76, P<0.001, \kappa=0.679$.

Table 2 Numbers of cases with mixed DCIS grade

\begin{tabular}{lcccc}
\hline & \multicolumn{4}{c}{ Number of cases with mixed DCIS grades } \\
\cline { 2 - 5 } & $\mathbf{3 + 2 + \mathbf { I }}$ & $\mathbf{3 + 2}$ & $\mathbf{3 + 1}$ & $\mathbf{2 + 1}$ \\
\hline Original DCIS & $\mid$ & 7 & 1 & 4 \\
Recurrent DCIS & 1 & 5 & 0 & 1 \\
\hline
\end{tabular}


the original lesion was pure low-grade DCIS. There was only fair agreement between the highest grade of the original DCIS and the grade of the first detected recurrent invasive tumour, whether this was in the first or subsequent recurrence $(\kappa=0.241)$. As shown in Table 3, in 27 of the 55 cases (49\%) the grades were the same, in the rest the invasive grade was lower in 25 and higher in three. In 21 of the former the DCIS was grade 3, while the invasive carcinoma was grade 2 . Table 4 shows that the DCIS grade of the recurrent in situ carcinoma associated with the invasive carcinoma was related to the grade of the invasive carcinoma $(\kappa=0.513)$. The grade was the same in 30 of 46 cases (65\%). The invasive grade was higher in two patients and lower in 14. In 11 of the latter the DCIS was grade 3 and the invasive carcinoma was grade 2 .

The different components of the grade of the invasive carcinoma (tubule formation, nuclear pleomorphism and mitoses) were analysed separately in relation to the grade of both the original and the recurrent in situ carcinomas. Fair agreement was found between the original DCIS grade and the pleomorphism score $(\kappa=0.396)$ (Table 5), but not with either tubule formation or the mitotic score. The recurrent DCIS was related to all the three components, but most strongly to pleomorphism $(\kappa=0.485)$ (Table 6).

Table 3 Comparison of original DCIS grade with recurrent invasive grade

\begin{tabular}{lcccc}
\hline & \multicolumn{4}{c}{ Recurrent invasive grade $^{\mathbf{a}}$} \\
\cline { 2 - 5 } $\begin{array}{l}\text { Original DCIS grade } \\
\text { (highest) }\end{array}$ & $\mathbf{I}$ & $\mathbf{2}$ & $\mathbf{3}$ & Total \\
\hline 1 & 2 & 1 & 1 & 4 \\
2 & 3 & 5 & 1 & 9 \\
3 & 1 & 21 & 20 & 42 \\
Total & 6 & 27 & 22 & 55 \\
\hline
\end{tabular}

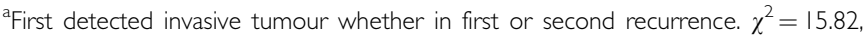
$P=0.003, \kappa=0.241$.

Table 4 Comparison of grades of DCIS and invasive carcinoma in recurrent lesions

\begin{tabular}{lcccc}
\hline & \multicolumn{4}{c}{ Associated recurrent invasive grade } \\
\cline { 2 - 5 } $\begin{array}{l}\text { Recurrent DCIS grade } \\
\text { (highest present) }\end{array}$ & $\mathbf{I}$ & $\mathbf{2}$ & $\mathbf{3}$ & Total \\
\hline 1 & 3 & 1 & 0 & 4 \\
2 & 3 & 8 & 1 & 12 \\
3 & 0 & 11 & 19 & 30 \\
Total & 6 & 20 & 20 & 46 \\
\hline
\end{tabular}

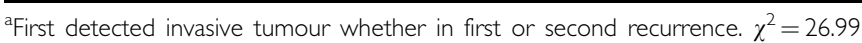
$P<0.001, \kappa=0.573$

Table 5 Comparison of original DCIS grade with pleomorphism score of recurrent invasive cancer

\begin{tabular}{lcccc}
\hline & \multicolumn{4}{l}{ Recurrent invasive grade pleomorphism score } \\
\cline { 2 - 5 } & $\mathbf{a}$ & & Total \\
$\begin{array}{l}\text { Original DCIS } \\
\text { grade (highest } \\
\text { present) }\end{array}$ & $\mathbf{1}$ & $\mathbf{2}$ & $\mathbf{3}$ & \\
\hline 1 & 1 & 1 & 2 & 4 \\
2 & 0 & 5 & 4 & 9 \\
3 & 0 & 6 & 36 & 42 \\
Total & 1 & 12 & 42 & 55 \\
\hline
\end{tabular}

${ }^{a}$ First detected invasive carcinoma whether in first or second recurrence. $\chi^{2}=21.12$ $P<0.001, \kappa=0.396$
Table 6 Comparison of recurrent DCIS grade with pleomorphism score of recurrent invasive cancer

\begin{tabular}{lcccc}
\hline & \multicolumn{4}{c}{$\begin{array}{c}\text { Associated recurrent invasive carcinoma } \\
\text { pleomorphism score }\end{array}$} \\
\cline { 2 - 5 } $\begin{array}{l}\text { Recurrent DCIS } \\
\text { grade (highest) }\end{array}$ & $\mathbf{4}$ & $\mathbf{2}$ & $\mathbf{3}$ & Total \\
\hline 1 & & & & \\
2 & 1 & 3 & 0 & 4 \\
3 & 0 & 6 & 7 & 13 \\
Total & 0 & 3 & 26 & 29 \\
\hline
\end{tabular}

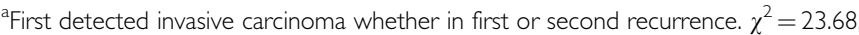
$P<0.001, \kappa=0.485$

Table 7 Comparison of the presence of necrosis in original and recurrent DCIS

\begin{tabular}{lrrrrc}
\hline & \multicolumn{5}{c}{ Necrosis recurrent DCIS } \\
\cline { 2 - 6 } Necrosis original DCIS & $\mathbf{0}$ & $\mathbf{I}$ & $\mathbf{2}$ & $\mathbf{3}$ & Total \\
\hline 0 & 15 & 6 & 5 & 4 & 30 \\
1 & 8 & 4 & 3 & 4 & 19 \\
2 & 1 & 2 & 6 & 5 & 14 \\
3 & 4 & 2 & 2 & 16 & 24 \\
Total & 28 & 14 & 16 & 29 & 87 \\
\hline
\end{tabular}

$\chi^{2}=28.04, P<0.001, \kappa=0.380$.

Table 8 Comparison of the degree of inflammation in original and recurrent DCIS

\begin{tabular}{lcccc}
\hline & \multicolumn{4}{c}{ Inflammation recurrent DCIS } \\
\cline { 2 - 5 } Inflammation original DCIS & $\mathbf{I}$ & $\mathbf{2}$ & $\mathbf{3}$ & Total \\
\hline 1 & 33 & 15 & 1 & 49 \\
2 & 16 & 9 & 1 & 26 \\
3 & 0 & 6 & 6 & 12 \\
Total & 49 & 30 & 8 & 87 \\
\hline
\end{tabular}

$\chi^{2}=34.26, P<0.001, \kappa=0.340$.

As shown in Tables 7 and 8, the scores for necrosis and inflammatory reaction within the original and recurrent DCIS were also associated $(\kappa=0.380$ and 0.340 , respectively). No such association was found for architectural pattern or periductal fibrosis. The number of cases with a mixed pattern made analysis of the former difficult.

In addition (data not shown), high-grade DCIS was associated with high scores for necrosis, inflammatory reaction and fibrosis, and also with the presence of a solid growth pattern, either alone, or in combination with other patterns. High scores for necrosis, periductal fibrosis and inflammation and the presence of a solid growth pattern, in the original DCIS, were also associated with high-grade recurrent DCIS and with the presence of invasion in the recurrence.

\section{DISCUSSION}

This study of tumours from 122 patients who developed relapse of DCIS or invasive progression shows strong agreement between the grade of initial DCIS and subsequent recurrent in situ lesions $(\kappa=0.679)$. In $84 \%$ of cases they were the same. There is also an association, although less strong, between the grade of initial DCIS and the grade of subsequent invasive recurrences $(\kappa=0.241)$. These findings add strength to the report of similar findings in a subsection $(n=31)$ of the cases included in the current study 
(Barnes et al, 2000). They also support the findings of the recent EORTC study on recurrent lesions from 116 patients (Bijker et al, 2001b). In the latter study, the DCIS was found to be the same in the $70 \%$ of initial and recurrent lesions, and in $53 \%$ of cases the grade of the recurrent invasive carcinoma was the same.

In our study, where the grade of the initial DCIS and that of either the recurrent DCIS or the recurrent invasive tumour were not the same, the grade of the subsequent tumour was more often lower than higher. In a significant number of cases the initial DCIS grade was 3, whereas the recurrent invasive tumour was grade 2 . A similar association was found with the recurrent DCIS associated with the invasive carcinoma. This association of grade 3 DCIS with grade 2 invasive carcinoma has been noted previously (Lampejo et al, 1994; Barnes et al, 2000). Of more importance was the finding in one case each of recurrent invasive carcinoma grade 2 and 3 following an initial low-grade DCIS lesion. Conversely, however, invasive grade 1 carcinomas followed three cases of intermediate grade DCIS and one of high-grade DCIS.

The criteria used for grading DCIS and invasive carcinomas are not the same. The grading system for DCIS used in this study was that used in the UK NHSBSP, which is based only on nuclear morphology (Sloane et al, 1998). The additional evaluation of tubule formation and number of mitoses in the grading of invasive carcinomas may well account for some of the discrepancies between DCIS grade and that of the invasive tumours. Indeed, a closer association was found between the nuclear pleomorphism score for the recurrent invasive carcinoma and the original DCIS grade ( $\kappa=0.396$ and 0.485 , respectively).

A possible reason for the lack of agreement between the original and recurrent lesions is that the second lesion is a new primary tumour rather than a recurrence of the previous one. One way to try to evaluate this is to see whether or not the lesions arose at the same site. It was difficult in many cases to ascertain the site of recurrent lesions in the breast. Where this information could be found, it was in the same quadrant in the majority of cases $(>80 \%)$. In the EORTC study, $11 \%$ of recurrences occurred in a different quadrant from the original lesion, but this was not found to affect the level of agreement (Bijker et al, 2001b).

The EORTC study also evaluated the immunohistochemical profile of the initial and subsequent DCIS and found $63 \%$ agreement with staining for oestrogen and progesterone receptors, p53 and HER2/neu oncoprotein (Bijker et al, 2001b). Another study has looked at the chromosomal alterations of in situ carcinomas and recurrent lesions using comparative genome hybridisation (CGH). A high level of agreement in 18 of 20 cases was found, although the mean number of CGH changes was higher in the recurrent than the initial lesions (Waldman et al, 2000). Both of these studies add strength to the theory that the recurrent lesions following excision of DCIS are closely related to the original disease and almost certainly represent re-growth of residual tumour. This emphasises the importance of clear margins at the time of initial surgery.

The cases for this study were selected on the basis that the DCIS had recurred and inevitably included a large proportion of highgrade DCIS and only eight low-grade lesions. Most previous studies of recurrence following treatment of DCIS have found a higher incidence of both DCIS and invasive carcinoma following high-grade DCIS, at least in the short term (Lagios et al, 1989; Schwartz et al, 1992; Bellamy et al, 1993; Solin et al, 1993; Sneige et al, 1995; Silverstein et al, 1996; Fisher et al, 1999). In the EORTC study, although the risk of DCIS recurrence was found to be significantly related to the initial histology, the risk of invasive recurrence was not (Bijker et al, 2001a). However, patients with poorly differentiated DCIS had a significantly higher risk of developing distant metastases and of death, which must reflect the higher grade of invasive carcinomas associated with this type of DCIS. Furthermore, within well-differentiated DCIS, those with a clinging or micropapillary pattern had significantly lower rates of recurrence than those with a cribriform pattern.

The findings in the current study that high-grade DCIS is associated with high scores for periductal necrosis, fibrosis and inflammatory reaction and with the presence of a solid growth pattern is to be expected. An association between the scores for these features in the original DCIS and with the occurrence of high-grade DCIS or the presence of invasion in recurrent lesions is also to be anticipated.

The number of cases of recurrent invasive carcinoma in this study is small, particularly following low-grade DCIS. As recurrence following low-grade DCIS may occur long after diagnosis of the original lesion, long-term follow-up may be necessary to detect such lesions (Page et al, 1995; Solin et al, 1995). Although this study shows only fair agreement between the grade of the original DCIS and that of the subsequent invasive tumour, there is no suggestion in this or the EORTC study that low-grade lesions often progress to a higher grade. This is in agreement with the consistency in malignancy grade, which has been found in studies of primary invasive mammary carcinoma when compared to both local and distant recurrences (Hitchcock et al, 1989; Millis et al, 1998).

There is growing evidence that low-grade and high-grade carcinomas are two distinctly different entities. Immunohistochemical studies of a range of markers show that they have different profiles. Both in situ and invasive low-grade lesions express oestrogen and progesterone receptors, but are usually negative for oncoproteins (HER/neu and p53). High-grade lesions exhibit the reverse (Hitchcock et al, 1989; Gullick et al, 1991; Barnes et al, 1993; Millis et al, 1996; Ellis et al, 1999). Molecular genetic studies find that low-grade lesions often demonstrate $16 \mathrm{q}$ deletions, while high-grade lesions have allelic losses of more chromosomal areas frequently including $1 \mathrm{p}, 1 \mathrm{q}, 6 \mathrm{q}, 9 \mathrm{p}, 11 \mathrm{p}, 13 \mathrm{q}$ and $17 \mathrm{q}$. Intermediate grade DCIS tends to show combinations of the patterns associated with low- and high-grade lesions (Buerger et al, 1999a and b; Ellis et al, 1999; Roylance et al, 2002).

This study adds weight to the idea that recurrence following treatment of DCIS represents re-growth of residual disease. It also supports the concept that the grade of mammary carcinoma does not progress with time. Further studies with longer follow-up and, if possible, greater numbers of low-grade tumours are needed finally to answer the question. Owing to the natural history of low-grade DCIS, it may be necessary to combine the results from many centres in order to obtain sufficient numbers of recurrent lesions for analysis.

\section{ACKNOWLEDGEMENTS}

We thank the clinicians at the Royal Marsden Hospitals, Guy's Hospital, Nottingham City Hospital and The Royal Liverpool Hospital for making available the clinical details of the patients in this study. They are also indebted to Professor Barry Gusterson and the late Professor John Sloane for identifying some of the cases.

\section{REFERENCES}

Barnes DM, Dublin EA, Fisher CJ, Levison DA, Millis RR (1993) Immunohistochemical detection of $\mathrm{p} 53$ protein in mammary carcinoma: an important new independent indicator of prognosis? Hum Pathol 24: $469-476$
Barnes P, Wahedna Y, Pinder SE, Lee AHS, Geraghty J, Blamey RW, Ellis IO (2000) Histological features of recurrent disease following treatment for ductal carcinoma in situ of the breast (DCIS). J Pathol 19(S): $5 \mathrm{~A}$ 
Bellamy COC, McDonald C, Salter DM, Chetty U, Anderson TJ (1993) Noninvasive ductal carcinoma of the breast: the relevance of histologic categorization. Hum Pathol 24: 16-23

Bijker N, Peterse JL, Duchateau L, Julien J-P, Fentiman IS, Duval C, Di Palma S, Simony-Lafontaine J, de Mascarel I, van de Vijver MJ (2001a) Risk factors for recurrence and metastasis after breast-conserving therapy for ductal carcinoma-in-situ: analysis of European Organization for Research and Treatment of Cancer Trial 10853. J Clin Oncol 19: $2263-2271$

Bijker N, Peterse JL, Duchateau L, Robanus-Maandag EC, Bosch CAJ, DuvaI C, Pilotti S, van de Vijver MJ (2001b) Histological type and marker expression of the primary tumour compared with its local recurrence after breast-conserving therapy for ductal carcinoma in situ. Br J Cancer 84: $539-544$

Buerger H, Otterbach F, Simon R, Poremba C, Diallo R, Decker T, Riethdorf L, Brinkschmidt C, Dockhorn-Dworniczak B, Boecker W (1999a) Comparative genomic hybridization of ductal carcinoma in situ of the breast-evidence of multiple genetic pathways. J Pathol 187: 396-402

Buerger H, Otterbach F, Simon R, Schafer KL, Poremba C, Diallo R, Brinkschmidt C, Dockhorn-Dworniczak B, Boecker W (1999b) Different genetic pathways in the evolution of invasive breast cancer are associated with distinct morphological subtypes. J Pathol 189: 521-526

Douglas-Jones AG, Logan J, Morgan JM, Johnson R, Williams R (2002) Effect of margins of excision on recurrence after local excision of ductal carcinoma in situ of the breast. J Clin Pathol 55: 581-586

Ellis IO, Pinder SE, Lee AHS, Elston CW (1999) A critical appraisal of existing classification systems of epithelial hyperplasia and in situ neoplasia of the breast with proposals for future methods of categorization: where are we going? Semin Diag Pathol 16: 202-208

Elston CW, Ellis IO (1991) Pathological prognostic factors in breast cancer I. The value of histological grade in breast cancer: experience from a large study with long-term follow-up. Histopathology 19: 403-410

Fisher ER, Dignam J, Tan-Chiu E, Costantino J, Fisher B, Paik S, Wolmark $\mathrm{N}$, for the National Surgical Breast and Bowel Project (NSABP) Collaborating Investigators (1999) Pathologic findings from the National Surgical Adjuvant Breast Project (NSABP) eight-year update of Protocol B-17: intraductal carcinoma. Cancer 86: 429-438

Goldstein NS, Murphy T (1996) Intraductal carcinoma associated with invasive carcinoma of the breast: a comparison of the two lesions with implications for intraductal carcinoma classification systems. Am J Clin Pathol 106: $312-318$

Gullick WJ, Love SB, Wright S, Barnes DM, Gusterson B, Harris AL, Altman DG (1991) c-erbB-2 protein overexpression in breast cancer is a risk factor in patients with involved and uninvolved lymph nodes. $\mathrm{Br}$ J Cancer 63: $434-438$

Gupta SK, Douglas-Jones AG, Fenn N, Morgan JM, Mansel RE (1997) The clinical behaviour of breast carcinoma is probably determined at the preinvasive stage (ductal carcinoma in situ). Cancer 80: 1740-1745

Hitchcock A, Ellis IO, Robertson JF, Gilmour A, Bell J, Elston CW, Blamey RW (1989) An observation of DNA ploidy, histological grade and immunoreactivity for tumour-related antigens in primary and metastatic breast carcinoma. J Pathol 159: 129-134

Holland R, Peterse JL, Millis RR, Eusebi V, Faverly D, van de Vijver MJ, Zafrani B (1994) Ductal carcinoma to situ: a proposal for a new classification. Semin Diag Pathol 11: 167-180

Julien J-P, Bijker N, Fentiman IS, Peterse JL, Delledonne V, Rouanet P, Avril A, Sylvester R, Mignolet FH, Van Dongen JA (2000) The role of radiotherapy in breast conserving treatment for ductal carcinoma in situ: first results of EORTC randomised phase III trial 10853. Lancet 355: 528-533

Lagios MD, Margolin FR, Westdahl PR, Rose MR (1989) Mammographically detected duct carcinoma in situ. Frequency of local recurrence following tylectomy and prognostic effect of nuclear grade on local recurrence. Cancer 63: 618-624

Lampejo OT, Barnes DM, Smith P, Millis RR (1994) Evaluation of infiltrating ductal carcinomas with a DCIS component: correlation of the histological type of the in situ component with grade of the infiltrating component. Semin Diag Pathol 11: 215-222

Millis RR, Barnes DM, Lampejo OT, Egan MK, Smith P (1998) Tumour grade does not change between primary and recurrent mammary carcinoma. Eur J Cancer 34: 548-555
Millis RR, Bobrow LG, Barnes DM (1996) Immunohistochemical evaluation of biological markers in mammary carcinoma in situ: correlation with morphological features and recently proposed schemes for histological classification. Breast 5: $113-122$

Page DL, Dupont WD, Rogers LW, Jensen RA, Schuyler PA (1995) Continued local recurrence of carcinoma $15-25$ years after a diagnosis of low grade ductal carcinoma in situ of the breast treated only by biopsy. Cancer 76: 11197 - 11199

Poller D, Ellis I (1995) Ductal carcinoma in situ (DCIS) of the breast. In Progress in Pathology, Kirkham N, Lemoine N (eds) Vol. 2, pp 47-87. Edinburgh: Churchill Livingstone

Poller DN, Silverstein MJ, Galea M, Locker AP, Elston CW, Blamey RW, Ellis IO (1994) Ductal carcinoma in situ of the breast: a proposal for a new simplified histological classification, association between cellular proliferation and c-erb B-2 protein expression. Mod Pathol 7: $257-262$

Roylance R, Gorman P, Hanby A, Tomlinson I (2002) Allelic imbalance analysis of chromosome $16 \mathrm{q}$ shows that grade I and grade III invasive ductal breast cancers follow different genetic pathways. J Pathol 196: $32-36$

Schwartz GF, Finkel GC, Garcia JC, Patchefsky AS (1992) Subclinical ductal carcinoma in situ of the breast: treatment by local excision and surveillance alone. Cancer 70: 2468-2474

Scott MA, Lagios MD, Axelsson K, Rogers LW, Anderson TJ, Page DL (1997) Ductal carcinoma in situ of the breast: reproducibility of histological subtype analysis. Hum Pathol 28: 967-973

Silverstein MJ, Lagios MD, Craig PH, Waisman JR, Lewinsky BS, Colburn WJ, Poller DN (1996) A prognostic index for ductal carcinoma in situ of the breast. Cancer 77: 2267-2274

Silverstein MJ, Lagios MD, Groshen S, Waisman JR, Lewinsky BS, Martino S, Gamagami P, Colburn WJ (1999) The influence of margin width on local control of ductal carcinoma in situ of the breast. $N$ Engl J Med 340: $1455-1461$

Silverstein MJ, Poller DN, Waisman JR, Colburn WJ, Barth A, Gierson D, Lewinsky B, Gamagami P, Slamon DJ (1995) Prognostic classification of breast ductal carcinoma in situ. Lancet 345: 1154-1157

Sloane JP, Amendoeira I, Apostolikas N, Bellocq JP, Bianchi S, Boecker W, Bussolati G, Coleman D, Connolly CE, Dervan P, Eusebi V, De Miguel C, Drijkoningen M, Elston CW, Faverley D, Gad A, Jacquemier J, Lacerda M, Martinez-Penuela J, Munt C, Peterse JL, Rank F, Sylvan M, Tsakraklides V, Zafrani B (1998) Consistency achieved by 23 European pathologists in categorizing ductal carcinoma in situ of the breast using five classifications. European Commission Working Group on Breast Screening Pathology. Hum Pathol 29: 1056-1062

Sneige N, McNeese MD, Atkinson EN, Ames FC, Kemp B, Sahin A, Ayala AG (1995) Ductal carcinoma in situ treated with lumpectomy and irradiation: histopathological analysis of 49 specimens with emphasis on risk factors and long term results. Hum Pathol 26: $642-649$

Solin LJ, Kurtz J, Forquet A, Amalric R, Recht A, Bornstein BA, Kuske R, Taylor M, Barrett W, Fowble B, Haffty B, Schultz DR, Yeh I-T, McCormick B, McNeese M (1995) Fifteen-year results of the treatment of breast-conserving surgery and definitive breast irradiation for the treatment of ductal carcinoma in situ of the breast. J Clin Oncol 14: $754-763$

Solin LJ, Yeh I-T, Kurtz J, Fourquet A, Recht A, Kuske R, McCormick B, Cross MA, Schultz DJ, Amalric R, LiVolsi VA, Kowalyshyn MJ, Torhorst J, Jacquemier J, Westermann CD, Mazoujian G, Zafrani B, Rosen PP, Goodman RL, Fowble BL (1993) Ductal carcinoma in situ (intraductal carcinoma) of the breast treated with breast-conserving surgery and definitive irradiation: correlation of pathologic parameters with outcome of treatment. Cancer 71: 2532-2542

UK Coordinating Committee on Cancer Research (UKCCCR) Ductal Carcinoma in situ (DCIS) Working Party (2003) The UK, Australian and New Zealand randomised trial comparing radiotherapy and tamoxifen in women with completely excised ductal carcinoma in situ of the breast. Lancet 362: 95-102

Waldman FM, DeVries S, Chew KL, Moore DH, Kerlikowske K, Ljung B-M (2000) Chromosomal alterations in ductal carcinomas in situ and their in situ recurrences. J Natl Cancer Inst 92: 313-320 